\title{
Sharp well-posedness of the Cauchy problem for a generalized Ostrovsky equation with positive dispersion
}

\author{
Junfang Wang ${ }^{1 *}$ (D) and Zongmin Wang ${ }^{2}$
}

"Correspondence:
wangjunfang0309@126.com
1School of Mathematics and
Statistics, Lanzhou University,
LanZhou, Gansu 730000, P.R. China
Full list of author information is
available at the end of the article

available at the end of the article

\begin{abstract}
The goal of this paper is two-fold. Firstly, by using the Fourier restriction norm method and the fixed point theorem, we prove that the Cauchy problem for a generalized Ostrovsky equation$$
\partial_{x}\left(u_{t}-\beta \partial_{x}^{3} u+\frac{1}{3} \partial_{x}\left(u^{3}\right)\right)-\gamma u=0, \quad \beta>0, \gamma>0,
$$

is locally well-posed in $H^{s}(\mathbf{R})$ with $s \geq \frac{1}{4}$. Secondly, we prove that the Cauchy problem for a generalized Ostrovsky equation is not well-posed in $H^{s}(\mathbf{R})$ with $s<\frac{1}{4}$ in the sense that the solution map is $C^{3}$.
\end{abstract}

MSC: $35 G 25$

Keywords: generalized Ostrovsky equation with positive dispersion; Cauchy problem; sharp well-posedness

\section{Introduction}

In this paper, we are concerned with the Cauchy problem for a generalized Ostrovsky equation with positive dispersion,

$$
\partial_{x}\left(u_{t}-\beta \partial_{x}^{3} u+\frac{1}{3} \partial_{x}\left(u^{3}\right)\right)-\gamma u=0, \quad \gamma>0, \beta \in \mathbf{R} .
$$

Here $u(x, t)$ represents the free surface of the liquid and the parameter $\gamma>0$ measures the effect of rotation. (1.1) describes the propagation of internal waves of even modes in the ocean; for instance, see the work of Galkin and Stepanyants [1], Leonov [2], and Shrira [3, 4]. The parameter $\beta$ determines the type of dispersion, more precisely, when $\beta<0,(1.1)$ denotes the generalized Ostrovsky equation with negative dispersion; when $\beta>0,(1.1)$ denotes the generalized Ostrovsky equation with positive dispersion.

When $\gamma=0$, (1.1) reduces to the modified Korteweg-de Vries equation which has been investigated by many authors; for instance, see [5-11]. Kenig et al. [9] proved that the Cauchy problem for the modified KdV equation is locally well-posed in $H^{s}(\mathbf{R})$ with $s \geq \frac{1}{4}$. Kenig et al. [10] proved that the Cauchy problem for the modified KdV equation is illposed in $H^{s}(\mathbf{R})$ with $s<\frac{1}{4}$. Colliander et al. [6] proved that the Cauchy problem for the

(c) The Author(s) 2017. This article is distributed under the terms of the Creative Commons Attribution 4.0 International License (http://creativecommons.org/licenses/by/4.0/), which permits unrestricted use, distribution, and reproduction in any medium, provided you give appropriate credit to the original author(s) and the source, provide a link to the Creative Commons license, and indicate if changes were made. 
modified KdV equation is globally well-posed in $H^{s}(\mathbf{R})$ with $s>\frac{1}{4}$ and globally well-posed in $H^{s}(\mathbf{T})$ with $s \geq \frac{1}{2}$. Guo [7] and Kishimoto [11] proved that the modified KdV equation is globally well-posed in $H^{\frac{1}{4}}(\mathbf{R})$ with the aid of the $I$ method and some new spaces.

Now we give a brief review of the Ostrovsky equation,

$$
u_{t}-\beta \partial_{x}^{3} u+\frac{1}{3} \partial_{x}\left(u^{2}\right)-\gamma \partial_{x}^{-1} u=0, \quad \gamma>0 .
$$

Equation (1.2) was proposed by Ostrovsky in [12] as a model for weakly nonlinear long waves in a rotating liquid, by taking into account the Coriolis force, to describe the propagation of surface waves in the ocean in a rotating frame of reference. The parameter $\beta$ determines the type of dispersion, more precisely, $\beta<0$ (negative dispersion) for surface and internal waves in the ocean or surface waves in a shallow channel with an uneven bottom and $\beta>0$ (positive dispersion) for capillary waves on the surface of liquid or for oblique magneto-acoustic waves in plasma [1,13-15]. Some authors have investigated the stability of the solitary waves or soliton solutions of (1.2); for instance, see [16-18].

Many people have studied the Cauchy problem for (1.2), for instance, see [17, 19-30]. The result of $[23,25,31]$ showed that $s=-\frac{3}{4}$ is the critical regularity index for (1.2). Coclite and di Ruvo $[32,33]$ have investigated the convergence of the Ostrovsky equation to the Ostrovsky-Hunter one and the dispersive and diffusive limits for Ostrovsky-Hunter type equation. Recently, Li et al. [34] proved that the Cauchy problem for the Ostrovsky equation with negative dispersion is locally well-posed in $H^{-\frac{3}{4}}(\mathbf{R})$.

Levandosky and Liu [16] studied the stability of solitary waves of the generalized Ostrovsky equation,

$$
\left[u_{t}-\beta u_{x x x}+(f(u))_{x}\right]_{x}=\gamma u, \quad x \in \mathbf{R},
$$

where $f$ is a $C^{2}$ function which is homogeneous of degree $p \geq 2$ in the sense that it satisfies $s f^{\prime}(s)=p f(s)$. Levandosky [18] studied the stability of ground state solitary waves of (1.4) with homogeneous nonlinearities of the form $f(u)=c_{1}|u|^{p}+c_{2}|u|^{p-1} u, c_{1}, c_{2} \in \mathbf{R}, p \geq 2$.

Equation (1.1) can be written in the following form:

$$
u_{t}-\beta \partial_{x}^{3} u+\frac{1}{3} \partial_{x}\left(u^{3}\right)-\gamma \partial_{x}^{-1} u=0 .
$$

Let $w(x, t)=\beta^{-\frac{1}{2}} u\left(x, \beta^{-1} t\right)$, then $w(x, t)$ is the solution to

$$
w_{t}-w_{x x x}+\frac{1}{3} \partial_{x}\left(w^{3}\right)-\gamma \beta^{-1} w=0 .
$$

Without loss of generality, we can assume that $\beta=\gamma=1$.

Motivated by [35], firstly, by using the $X_{s, b}$ spaces introduced by [36-40] and developed in $[8,41,42]$ and the Strichartz estimates established in $[19,43]$, we prove that (1.3) with initial data

$$
u(x, 0)=u_{0}(x)
$$

is locally well-posed in $H^{s}(\mathbf{R})$ with $s \geq \frac{1}{4}, \beta>0, \gamma>0$; secondly, we prove that the problems (1.3), (1.5) are not quantitatively well-posed in $H^{s}(\mathbf{R})$ with $s<\frac{1}{4}, \beta \neq 0, \gamma>0$. Thus, our result is sharp. 
We introduce some notations before giving the main result. Throughout this paper, we assume that $C$ is a positive constant which may vary from line to line and $0<\epsilon<10^{-4}$. $A \sim B$ means that $|B| \leq|A| \leq 4|B| . A \gg B$ means that $|A|>4|B| . \psi(t)$ is a smooth function supported in $[-1,2]$ and equals 1 in $[-1,1]$. We assume that $\mathcal{F} u$ is the Fourier transformation of $u$ with respect to both space and time variables and $\mathcal{F}^{-1} u$ is the inverse transformation of $u$ with respect to both space and time variables, while $\mathcal{F}_{x} u$ denotes the Fourier transformation of $u$ with respect to the space variable and $\mathcal{F}_{x}^{-1} u$ denotes the inverse transformation of $u$ with respect to the space variable. Let $I \subset \mathbf{R}, \chi_{I}(x)=1$ if $x \in I ; \chi_{I}(x)=0$ if $x$ does not belong to $I$. Let

$$
\langle\cdot\rangle=1+|\cdot|, \quad \phi(\xi)=\xi^{3}+\frac{1}{\xi}, \quad \sigma=\tau+\phi(\xi), \quad \sigma_{j}=\tau_{j}+\phi\left(\xi_{j}\right) \quad(j=1,2,3) .
$$

The space $X_{s, b}$ is defined by

$$
X_{s, b}=\left\{u \in \mathcal{S}^{\prime}\left(\mathbf{R}^{2}\right):\|u\|_{X_{s, b}}=\left\|\langle\xi\rangle^{s}\langle\tau+\phi(\xi)\rangle^{b} \mathcal{F} u(\xi, \tau)\right\|_{L_{\tau \xi}^{2}\left(\mathbf{R}^{2}\right)}<\infty\right\} .
$$

The space $X_{s, b}^{T}$ denotes the restriction of $X_{s, b}$ onto the finite time interval $[-T, T]$ and is equipped with the norm

$$
\|u\|_{X_{s, b}^{T}}=\inf \left\{\|w\|_{X_{s, b}}: w \in X_{s, b}, u(t)=w(t) \text { for }-T \leq t \leq T\right\} .
$$

The main results of this paper are as follows.

Theorem 1.1 Let $s \geq \frac{1}{4}$ and $\beta>0$ and $\gamma>0$. Then the problems (1.4), (1.5) are locally wellposed in $H^{s}(\mathbf{R})$. More precisely, for $u_{0} \in H^{s}(\mathbf{R})$, there exist a $T>0$ and a unique solution $u \in C\left([-T, T] ; H^{s}(\mathbf{R})\right)$.

Remark 1 The result of Theorem 1.1 is optimal in the sense of Theorem 1.2.

Theorem 1.2 Let $s<\frac{1}{4}$ and $\beta>0$ and $\gamma>0$. Then the problems (1.4), (1.5) are not wellposed in $H^{s}(\mathbf{R})$ in the sense that the solution map is $C^{3}$.

The rest of the paper is arranged as follows. In Section 2, we give some preliminaries. In Section 3, we establish a trilinear estimate. In Section 4, we prove Theorem 1.1. In Section 5, we prove Theorem 1.2.

\section{Preliminaries}

In this section, we give Lemmas 2.1-2.4.

Lemma 2.1 Let $0<\epsilon<\frac{1}{10^{8}}$ and $\mathcal{F}\left(P^{a} f\right)(\xi)=\chi_{\{|\xi| \geq a\}}(\xi) \mathcal{F} f(\xi)$ with $a \geq 2$ and $\mathcal{F}\left(D_{x}^{b} f\right)(\xi)=$ $|\xi|^{b} \mathcal{F} f(\xi)$ with $b \in \mathbf{R}$. Then we have

$$
\begin{aligned}
& \|u\|_{L_{x t}^{6}} \leq C\|u\|_{X_{0, \frac{1}{2}+\epsilon}}, \\
& \left\|D_{x}^{\frac{1}{6}} P^{a} u\right\|_{L_{x t}^{6}} \leq C\|u\|_{X_{0, \frac{1}{2}+\epsilon}}, \\
& \|u\|_{L_{x t}^{4}} \leq C\|u\|_{X_{0, \frac{3}{4}\left(\frac{1}{2}+\epsilon\right)}} \cdot
\end{aligned}
$$


For the proof of Lemma 2.1, we refer the reader to (2.27) and (2.21) of [19].

Lemma 2.2 $\operatorname{Let} \phi(\xi)=\xi^{3}+\frac{1}{\xi}$ and

$$
\mathcal{F}\left(I^{s}(u, v)\right)(\xi, \tau)=\int_{\substack{\xi=\xi_{1}+\xi_{2} \\ \tau=\tau_{1}+\tau_{2}}}\left|\phi^{\prime}\left(\xi_{1}\right)-\phi^{\prime}\left(\xi_{2}\right)\right|^{s} \mathcal{F} u_{1}\left(\xi_{1}, \tau_{1}\right) \mathcal{F} u_{2}\left(\xi_{2}, \tau_{2}\right) d \xi_{1} d \tau_{1}
$$

Then we have

$$
\left\|I^{\frac{1}{2}}\left(u_{1}, u_{2}\right)\right\|_{L_{x t}^{2}} \leq C \prod_{j=1}^{2}\left\|u_{j}\right\|_{0, \frac{1}{2}+\epsilon}
$$

For the proof of Lemma 2.2, we refer the reader to Lemma 2.5 of [43].

Lemma 2.3 Let $T \in(0,1)$ and $b \in\left(\frac{1}{2}, \frac{3}{2}\right)$. Then, for $s \in \mathbf{R}$ and $\theta \in\left[0, \frac{3}{2}-b\right)$, we have

$$
\begin{aligned}
& \left\|\eta_{T}(t) S(t) \phi\right\|_{X_{s, b}\left(\mathbf{R}^{2}\right)} \leq C T^{\frac{1}{2}-b}\|\phi\|_{H^{s}(\mathbf{R})}, \\
& \left\|\eta_{T}(t) \int_{0}^{t} S(t-\tau) F(\tau) d \tau\right\|_{X_{s, b}\left(\mathbf{R}^{2}\right)} \leq C T^{\theta}\|F\|_{X_{s, b-1+\theta}\left(\mathbf{R}^{2}\right)}
\end{aligned}
$$

For the proof of Lemma 2.3, we refer the reader to $[8,39,44]$.

Lemma 2.4 Let $a_{j} \in \mathbf{R}(j=1,2,3)$ and $\prod_{j=1}^{3} a_{j} \neq 0$. Then we have

$$
\begin{aligned}
& \left(\sum_{j=1}^{3} a_{j}\right)^{3}+\frac{1}{\sum_{j=1}^{3} a_{j}}-\sum_{j=1}^{3}\left(a_{j}^{3}+\frac{1}{a_{j}}\right) \\
& \quad=3\left(a_{1}+a_{2}\right)\left(a_{1}+a_{3}\right)\left(a_{2}+a_{3}\right)\left[1-\frac{1}{3 \prod_{j=1}^{3} a_{j}\left(\sum_{j=1}^{3} a_{j}\right)}\right]
\end{aligned}
$$

Proof By using the following two identities:

$$
\begin{aligned}
& \left(\sum_{j=1}^{3} a_{j}\right)^{3}-\left(\sum_{j=1}^{3} a_{j}^{3}\right)=3\left(a_{1}+a_{2}\right)\left(a_{1}+a_{3}\right)\left(a_{2}+a_{3}\right), \\
& \left(\sum_{j=1}^{3} a_{j}\right)\left(a_{1} a_{2}+a_{1} a_{3}+a_{2} a_{3}\right)-\prod_{j=1}^{3} a_{j}=\left(a_{1}+a_{2}\right)\left(a_{1}+a_{3}\right)\left(a_{2}+a_{3}\right),
\end{aligned}
$$


which can be found in [6], we have

$$
\begin{aligned}
& \left(\sum_{j=1}^{3} a_{j}\right)^{3}+\frac{1}{\sum_{j=1}^{3} a_{j}}-\sum_{j=1}^{3}\left(a_{j}^{3}+\frac{1}{a_{j}}\right) \\
& =\left(\sum_{j=1}^{3} a_{j}\right)^{3}-\sum_{j=1}^{3} a_{j}^{3}-\left[\sum_{j=1}^{3} \frac{1}{a_{j}}-\frac{1}{\sum_{j=1}^{3} a_{j}}\right] \\
& =3\left(a_{1}+a_{2}\right)\left(a_{1}+a_{3}\right)\left(a_{2}+a_{3}\right)-\left[\frac{\left(\sum_{j=1}^{3} a_{j}\right)\left(a_{1} a_{2}+a_{1} a_{3}+a_{2} a_{3}\right)+\prod_{j=1}^{3} a_{j}}{\prod_{j=1}^{3} a_{j}\left(\sum_{j=1}^{3} a_{j}\right)}\right] \\
& =3\left(a_{1}+a_{2}\right)\left(a_{1}+a_{3}\right)\left(a_{2}+a_{3}\right)-\left[\frac{\left(a_{1}+a_{2}\right)\left(a_{1}+a_{3}\right)\left(a_{2}+a_{3}\right)}{\prod_{j=1}^{3} a_{j}\left(\sum_{j=1}^{3} a_{j}\right)}\right] \\
& =3\left(a_{1}+a_{2}\right)\left(a_{1}+a_{3}\right)\left(a_{2}+a_{3}\right)\left[1-\frac{1}{3 \prod_{j=1}^{3} a_{j}\left(\sum_{j=1}^{3} a_{j}\right)}\right]
\end{aligned}
$$

Thus, (2.5) is valid.

This ends the proof of Lemma 2.4.

\section{The trilinear estimate}

In this section, by using Lemmas 2.1-2.2, we give the proof of Lemma 3.1.

Lemma 3.1 Let $u_{j} \in X_{s, \frac{1}{2}+\epsilon}$ with $s \geq \frac{1}{4}$ and $j=1,2,3$. Then we have

$$
\left\|\partial_{x}\left(\prod_{j=1}^{3} u_{j}\right)\right\|_{X_{s,-\frac{1}{2}+2 \epsilon}} \leq C \prod_{j=1}^{3}\left\|u_{j}\right\|_{X_{s, \frac{1}{2}+\epsilon}} .
$$

Proof To prove (3.1), by duality, it suffices to prove that

$$
\int_{\mathbf{R}^{2}} \bar{u}(x, t) \partial_{x}\left(\prod_{j=1}^{3} u_{j}\right) d x d t \leq C\left[\prod_{j=1}^{3}\left\|u_{j}\right\|_{X_{s, \frac{1}{2}+\epsilon}}\right]\|u\|_{X_{-s, \frac{1}{2}-2 \epsilon}} .
$$

Let

$$
\begin{aligned}
& F(\xi, \tau)=\langle\xi\rangle^{-s}\langle\sigma\rangle^{\frac{1}{2}-2 \epsilon} \mathcal{F} u(\xi, \tau), \\
& F_{j}\left(\xi_{j}, \tau_{j}\right)=\left\langle\xi_{j}\right\rangle^{s}\left\langle\sigma_{j}\right\rangle^{\frac{1}{2}+\epsilon} \mathcal{F} u_{j}\left(\xi_{j}, \tau_{j}\right) \quad(j=1,2,3) .
\end{aligned}
$$

To obtain (3.2), from (3.3), it suffices to prove that

$$
\begin{aligned}
& \int_{\mathbf{R}^{2}} \int_{\substack{\xi=\xi_{1}+\xi_{2}+\xi_{3} \\
\tau=\tau_{1}+\tau_{2}+\tau_{3}}} \frac{|\xi|\langle\xi\rangle^{s} F(\xi, \tau) \prod_{j=1}^{3} F_{j}\left(\xi_{j}, \tau_{j}\right)}{\langle\sigma\rangle^{\frac{1}{2}-2 \epsilon} \prod_{j=1}^{3}\left\langle\xi_{j}\right\rangle^{s}\left\langle\sigma_{j}\right\rangle^{\frac{1}{2}+\epsilon}} d \xi_{1} d \tau_{1} d \xi_{2} d \tau_{2} d \xi d \tau \\
& \quad \leq C\|F\|_{L_{\xi \tau}^{2}}\left(\prod_{j=1}^{3}\left\|F_{j}\right\|_{L_{\xi \tau}^{2}}\right) .
\end{aligned}
$$


Without loss of generality, by using the symmetry, we assume that $\left|\xi_{1}\right| \geq\left|\xi_{2}\right| \geq\left|\xi_{3}\right|$ and $F(\xi, \tau) \geq 0, F_{j}\left(\xi_{j}, \tau_{j}\right) \geq 0(j=1,2)$. We define

$$
\begin{aligned}
& \Omega_{1}=\left\{\left(\xi_{1}, \tau_{1}, \xi_{2}, \tau_{2}, \xi, \tau\right) \in \mathrm{R}^{6}, \xi=\sum_{j=1}^{3} \xi_{j}, \tau=\sum_{j=1}^{3} \tau_{j},\left|\xi_{3}\right| \leq\left|\xi_{2}\right| \leq\left|\xi_{1}\right| \leq 64\right\}, \\
& \Omega_{2}=\left\{\left(\xi_{1}, \tau_{1}, \xi_{2}, \tau_{2}, \xi, \tau\right) \in \mathrm{R}^{6}, \xi=\sum_{j=1}^{3} \xi_{j}, \tau=\sum_{j=1}^{3} \tau_{j},\left|\xi_{1}\right| \geq 64,\left|\xi_{1}\right| \geq 4\left|\xi_{2}\right|\right\}, \\
& \Omega_{3}=\left\{\left(\xi_{1}, \tau_{1}, \xi_{2}, \tau_{2}, \xi, \tau\right) \in \mathrm{R}^{6}, \xi=\sum_{j=1}^{3} \xi_{j}, \tau=\sum_{j=1}^{3} \tau_{j},\left|\xi_{1}\right| \geq 64,\left|\xi_{1}\right| \sim\left|\xi_{2}\right|,\left|\xi_{2}\right| \gg\left|\xi_{3}\right|\right\}, \\
& \Omega_{4}=\left\{\left(\xi_{1}, \tau_{1}, \xi_{2}, \tau_{2}, \xi, \tau\right) \in \mathrm{R}^{6}, \xi=\sum_{j=1}^{3} \xi_{j}, \tau=\sum_{j=1}^{3} \tau_{j},\left|\xi_{1}\right| \geq 64,\left|\xi_{1}\right| \sim\left|\xi_{2}\right| \sim\left|\xi_{3}\right|\right\} .
\end{aligned}
$$

Obviously, $\left\{\left(\xi_{1}, \tau_{1}, \xi_{2}, \tau_{2}, \xi, \tau\right) \in \mathrm{R}^{6}, \xi=\sum_{j=1}^{3} \xi_{j}, \tau=\sum_{j=1}^{3} \tau_{j},\left|\xi_{3}\right| \leq\left|\xi_{2}\right| \leq\left|\xi_{1}\right|\right\} \subset \bigcup_{j=1}^{4} \Omega_{j}$. Let

$$
K\left(\xi_{1}, \tau_{1}, \xi_{2}, \tau_{2}, \xi, \tau\right)=\frac{|\xi|\langle\xi\rangle^{s}}{\langle\sigma\rangle^{\frac{1}{2}-2 \epsilon} \prod_{j=1}^{3}\left\langle\sigma_{j}\right\rangle^{\frac{1}{2}+\epsilon}}
$$

and

$$
I=\int_{\mathbf{R}^{2}} \int_{\substack{\xi=\sum_{j=1}^{3} \xi_{j} \\ \tau=\sum_{j=1}^{3} \tau_{j}}} K\left(\xi_{1}, \tau_{1}, \xi_{2}, \tau_{2}, \xi, \tau\right) F(\xi, \tau) \prod_{j=1}^{3} F_{j}\left(\xi_{j}, \tau_{j}\right) d \xi_{1} d \tau_{1} d \xi_{2} d \tau_{2} d \xi d \tau
$$

(1) $\Omega_{1}$. In this subregion, we have

$$
K\left(\xi_{1}, \tau_{1}, \xi_{2}, \tau_{2}, \xi, \tau\right) \leq \frac{C}{\langle\sigma\rangle^{\frac{1}{2}-2 \epsilon} \prod_{j=1}^{3}\left\langle\sigma_{j}\right\rangle^{\frac{1}{2}+\epsilon}} .
$$

By using (3.6) and the Cauchy-Schwartz inequality and the Plancherel identity and the Hölder inequality as well as (2.1), we have

$$
\begin{aligned}
I & \leq C \int_{\mathbf{R}^{2}} \int_{\substack{\xi=\sum_{j=1}^{3} \xi_{j} \\
\tau=\sum_{j=1}^{3} \tau_{j}}} \frac{F(\xi, \tau) \prod_{j=1}^{3} F_{j}\left(\xi_{j}, \tau_{j}\right)}{\langle\sigma\rangle^{\frac{1}{2}-2 \epsilon} \prod_{j=1}^{3}\left\langle\sigma_{j}\right\rangle^{\frac{1}{2}+\epsilon}} d \xi_{1} d \tau_{1} d \xi_{2} d \tau_{2} d \xi d \tau \\
& \leq C\left\|\frac{F(\xi, \tau)}{\langle\sigma\rangle^{\frac{1}{2}-2 \epsilon}}\right\|_{L_{\xi \tau}^{2} \tau}\left\|\int_{\substack{\xi=\sum_{j=1}^{3} \xi_{j} \\
\tau=\sum_{j=1}^{3} \tau_{j}}} \frac{\prod_{j=1}^{3} F_{j}\left(\xi_{j}, \tau_{j}\right)}{\prod_{j=1}^{3}\left\langle\sigma_{j}\right\rangle^{\frac{1}{2}+\epsilon}} d \xi_{1} d \tau_{1} d \xi_{2} d \tau_{2}\right\|_{L_{\xi \tau}^{2}} \\
& \leq C\|F\|_{L_{\xi \tau}^{2}}\left(\prod_{j=1}^{3}\left\|\mathcal{F}^{-1}\left(\frac{F_{j}}{\left\langle\sigma_{j}\right\rangle^{\frac{1}{2}+\epsilon}}\right)\right\|_{L_{x t}^{6}}\right) \\
& \leq C\|F\|_{L_{\xi \tau}^{2}}\left(\prod_{j=1}^{3}\left\|F_{j}\right\|_{L_{\xi \tau}^{2}}\right) .
\end{aligned}
$$


(2) $\Omega_{2}$. In this subregion, since $\left|\phi^{\prime}\left(\xi_{1}\right)-\phi^{\prime}\left(\xi_{2}\right)\right|=3\left|\xi_{1}^{2}-\xi_{2}^{2}\right|\left|1+\frac{1}{3 \xi_{1}^{2} \xi_{2}^{2}}\right| \geq 3\left|\xi_{1}^{2}-\xi_{2}^{2}\right| \geq C|\xi|^{2}$ and $|\xi| \sim\left|\xi_{1}\right|$, we have

$$
\begin{aligned}
K\left(\xi_{1}, \tau_{1}, \xi_{2}, \tau_{2}, \xi, \tau\right) & \leq \frac{C|\xi|}{\langle\sigma\rangle^{\frac{1}{2}-2 \epsilon} \prod_{j=1}^{3}\left\langle\sigma_{j}\right\rangle^{\frac{1}{2}+\epsilon}} \\
& \leq C \frac{C\left|\xi_{1}^{2}-\xi_{2}^{2}\right|^{\frac{1}{2}}\left|1+\frac{1}{3 \xi_{1}^{2} \xi_{2}^{2}}\right|^{\frac{1}{2}}}{\langle\sigma\rangle^{\frac{1}{2}-2 \epsilon} \prod_{j=1}^{3}\left\langle\sigma_{j}\right\rangle^{\frac{1}{2}+\epsilon}}=\frac{C\left|\phi^{\prime}\left(\xi_{1}\right)-\phi^{\prime}\left(\xi_{2}\right)\right|^{\frac{1}{2}}}{\langle\sigma\rangle^{\frac{1}{2}-2 \epsilon} \prod_{j=1}^{3}\left\langle\sigma_{j}\right\rangle^{\frac{1}{2}+\epsilon}} .
\end{aligned}
$$

By using (3.7) and the Cauchy-Schwartz inequality and the Plancherel identity and the Hölder inequality as well as (2.3)-(2.4), since $\frac{3}{4}\left(\frac{1}{2}+\epsilon\right)<\frac{1}{2}-2 \epsilon$, we have

$$
\begin{aligned}
I \leq & C \int_{\mathbf{R}^{2}} \int_{\xi=\sum_{j=1}^{3} \xi_{j}} \frac{\left|\phi^{\prime}\left(\xi_{1}\right)-\phi^{\prime}\left(\xi_{2}\right)\right|^{\frac{1}{2}} F(\xi, \tau) \prod_{j=1}^{3} F_{j}\left(\xi_{j}, \tau_{j}\right)}{\langle\sigma\rangle^{\frac{1}{2}-2 \epsilon} \prod_{j=1}^{3}\left\langle\sigma_{j}\right\rangle^{\frac{1}{2}+\epsilon}} d \xi_{1} d \tau_{1} d \xi_{2} d \tau_{2} d \xi d \tau \\
\leq & C\left\|\mathcal{F}^{-1}\left(\frac{F}{\langle\sigma\rangle^{\frac{1}{2}-2 \epsilon}}\right)\right\|_{L_{x t}^{4}}\left\|I^{\frac{1}{2}}\left(\mathcal{F}^{-1}\left(\frac{F_{1}}{\left\langle\sigma_{1}\right\rangle^{\frac{1}{2}+\epsilon}}\right), \mathcal{F}^{-1}\left(\frac{F_{1}}{\left\langle\sigma_{2}\right\rangle^{\frac{1}{2}+\epsilon}}\right)\right)\right\|_{L_{x t}^{2}} \\
& \times\left\|\mathcal{F}^{-1}\left(\frac{F_{3}}{\left\langle\sigma_{3}\right\rangle^{\frac{1}{2}+\epsilon}}\right)\right\|_{L_{x t}^{4}} \\
\leq & C\|F\|_{L_{\xi \tau}^{2}}\left(\prod_{j=1}^{3}\left\|F_{j}\right\|_{L_{\xi \tau}^{2}}\right) .
\end{aligned}
$$

(3) $\Omega_{3}$. In this subregion, since $\left|\phi^{\prime}\left(\xi_{2}\right)-\phi^{\prime}\left(\xi_{3}\right)\right|=3\left|\xi_{2}^{2}-\xi_{3}^{2}\right|\left|1+\frac{1}{3 \xi_{2}^{2} \xi_{3}^{2}}\right| \geq 3\left|\xi_{2}^{2}-\xi_{3}^{2}\right| \geq C\left|\xi_{1}\right|^{2}$, we have

$$
\begin{aligned}
K\left(\xi_{1}, \tau_{1}, \xi_{2}, \tau_{2}, \xi, \tau\right) \leq & \frac{C\left|\xi_{1}\right|}{\langle\sigma\rangle^{\frac{1}{2}-2 \epsilon} \prod_{j=1}^{3}\left\langle\sigma_{j}\right\rangle^{\frac{1}{2}+\epsilon}} \\
& \leq C C \frac{C\left|\xi_{2}^{2}-\xi_{3}^{2}\right|^{\frac{1}{2}}\left|1+\frac{1}{3 \xi_{2}^{2} \xi_{3}^{2}}\right|^{\frac{1}{2}}}{\langle\sigma\rangle^{\frac{1}{2}-2 \epsilon} \prod_{j=1}^{3}\left\langle\sigma_{j}\right\rangle^{\frac{1}{2}+\epsilon}} \leq \frac{C\left|\phi^{\prime}\left(\xi_{2}\right)-\phi^{\prime}\left(\xi_{3}\right)\right|^{\frac{1}{2}}}{\langle\sigma\rangle^{\frac{1}{2}-2 \epsilon} \prod_{j=1}^{3}\left\langle\sigma_{j}\right\rangle^{\frac{1}{2}+\epsilon}} .
\end{aligned}
$$

By using (3.8) and the Cauchy-Schwartz inequality and the Plancherel identity and the Hölder inequality as well as (2.3)-(2.4), since $\frac{3}{4}\left(\frac{1}{2}+\epsilon\right)<\frac{1}{2}-2 \epsilon$, we have

$$
\begin{aligned}
I \leq & C \int_{\mathbf{R}^{2}} \int_{\substack{\xi=\sum_{j=1}^{3} \xi_{j} \\
\tau=\sum_{j=1}^{3} \tau_{j}}} \frac{\left|\phi^{\prime}\left(\xi_{2}\right)-\phi^{\prime}\left(\xi_{3}\right)\right|^{\frac{1}{2}} F(\xi, \tau) \prod_{j=1}^{3} F_{j}\left(\xi_{j}, \tau_{j}\right)}{\langle\sigma\rangle^{\frac{1}{2}-2 \epsilon} \prod_{j=1}^{3}\left\langle\sigma_{j}\right\rangle^{\frac{1}{2}+\epsilon}} d \xi_{1} d \tau_{1} d \xi_{2} d \tau_{2} d \xi d \tau \\
\leq & C\left\|\mathcal{F}^{-1}\left(\frac{F}{\langle\sigma\rangle^{\frac{1}{2}-2 \epsilon}}\right)\right\|_{L_{x t}^{4}}\left\|I^{\frac{1}{2}}\left(\mathcal{F}^{-1}\left(\frac{F_{2}}{\left\langle\sigma_{2}\right\rangle^{\frac{1}{2}+\epsilon}}\right), \mathcal{F}^{-1}\left(\frac{F_{3}}{\left\langle\sigma_{3}\right\rangle^{\frac{1}{2}+\epsilon}}\right)\right)\right\|_{L_{x t}^{2}} \\
& \times\left\|\mathcal{F}^{-1}\left(\frac{F_{1}}{\left\langle\sigma_{1}\right\rangle^{\frac{1}{2}+\epsilon}}\right)\right\|_{L_{x t}^{4}} \\
\leq & C\|F\|_{L_{\xi \tau}^{2}}\left(\prod_{j=1}^{3}\left\|F_{j}\right\|_{L_{\xi \tau}^{2}}\right) .
\end{aligned}
$$


(4) $\Omega_{4}$. In this subregion, since $s \geq \frac{1}{4}$ and $\left|\xi_{1}\right| \sim\left|\xi_{2}\right| \sim\left|\xi_{3}\right|$, we have

$$
K\left(\xi_{1}, \tau_{1}, \xi_{2}, \tau_{2}, \xi, \tau\right) \leq \frac{C\left|\xi_{1}\right|^{1-2 s}}{\langle\sigma\rangle^{\frac{1}{2}-2 \epsilon} \prod_{j=1}^{3}\left\langle\sigma_{j}\right\rangle^{\frac{1}{2}+\epsilon}} \leq \frac{C \prod_{j=1}^{3}\left|\xi_{j}\right|^{\frac{1}{6}}}{\langle\sigma\rangle^{\frac{1}{2}-2 \epsilon} \prod_{j=1}^{3}\left\langle\sigma_{j}\right\rangle^{\frac{1}{2}+\epsilon}} .
$$

By using (3.9) and the Cauchy-Schwartz inequality and the Plancherel identity and the Hölder inequality as well as $(2.2)$, since $\frac{3}{4}\left(\frac{1}{2}+\epsilon\right)<\frac{1}{2}-2 \epsilon$, we have

$$
\begin{aligned}
I & \leq C \int_{\mathbf{R}^{2}} \int_{\substack{\xi=\sum_{j=1}^{3} \xi_{j} \\
\tau=\sum_{j=1}^{3} \tau_{j}}} \frac{F(\xi, \tau) \prod_{j=1}^{3}\left|\xi_{j}\right|^{\frac{1}{6}} F_{j}\left(\xi_{j}, \tau_{j}\right)}{\langle\sigma\rangle^{\frac{1}{2}-2 \epsilon} \prod_{j=1}^{3}\left\langle\sigma_{j}\right\rangle^{\frac{1}{2}+\epsilon}} d \xi_{1} d \tau_{1} d \xi_{2} d \tau_{2} d \xi d \tau \\
& \leq C\left\|\frac{F}{\langle\sigma\rangle^{\frac{1}{2}-2 \epsilon}}\right\|_{L_{\xi \tau}^{2}}\left(\prod_{j=1}^{3}\left\|D_{x}^{\frac{1}{6}} P^{2} \mathcal{F}^{-1}\left(\frac{F_{j}}{\left\langle\sigma_{j}\right\rangle^{\frac{1}{2}+\epsilon}}\right)\right\|_{L_{x t}^{6}}\right) \\
& \leq C\|F\|_{L_{\xi \tau}^{2}}\left(\prod_{j=1}^{3}\left\|F_{j}\right\|_{L_{\xi \tau}^{2}}\right) .
\end{aligned}
$$

This completes the proof of Lemma 3.1.

\section{Proof of Theorem 1.1}

In this section, we use Lemmas 2.3, 3.1 to prove Theorem 1.1.

The solution to (1.3), (1.5) can be formally rewritten as follows:

$$
u(t)=e^{-t\left(-\partial_{x}^{3}-\partial_{x}^{-1}\right)} u_{0}+\frac{1}{3} \int_{0}^{t} e^{-(t-s)\left(-\partial_{x}^{3}-\partial_{x}^{-1}\right)} \partial_{x}\left(u^{3}\right) d s .
$$

We define

$$
\Phi(u)=\psi(t) e^{-t\left(-\partial_{x}^{3}-\partial_{x}^{-1}\right)} u_{0}+\frac{1}{3} \psi\left(\frac{t}{T}\right) \int_{0}^{t} e^{-(t-s)\left(-\partial_{x}^{3}-\partial_{x}^{-1}\right)} \partial_{x}\left(u^{3}\right) d s
$$

By taking advantaging of Lemmas $2.3,3.1$, we derive that

$$
\begin{aligned}
\|\Phi(u)\|_{X_{s, \frac{1}{2}+\epsilon}} & \leq C\left\|u_{0}\right\|_{H^{s}(\mathbf{R})}+C\left\|\psi\left(\frac{t}{T}\right) \int_{0}^{t} e^{-(t-s)\left(-\partial_{x}^{3}-\partial_{x}^{-1}\right)} \partial_{x}\left(u^{3}\right) d s\right\|_{X_{s, \frac{1}{2}+\epsilon}} \\
& \leq C\left\|u_{0}\right\|_{H^{s}(\mathbf{R})}+C T^{\epsilon}\left\|\partial_{x}\left(u^{3}\right) d s\right\|_{X_{s,-\frac{1}{2}+2 \epsilon}} \\
& \leq C\left\|u_{0}\right\|_{H^{s}(\mathbf{R})}+C T^{\epsilon}\|u\|_{X_{s, \frac{1}{2}+\epsilon}}^{3} .
\end{aligned}
$$

We define $B=\left\{u \in X_{s, \frac{1}{2}+\epsilon}:\|u\|_{X_{s, \frac{1}{2}+\epsilon}} \leq 2 C\left\|u_{0}\right\|_{H^{s}(\mathbf{R})}\right\}$. By using (4.3), by choosing $T$ sufficiently small such that $24 C^{3} T^{\epsilon}\left\|u_{0}\right\|_{H^{s}}^{2}<1$, we have

$$
\|\Phi(u)\|_{X_{s, \frac{1}{2}+\epsilon}} \leq C\left\|u_{0}\right\|_{H^{s}(\mathbf{R})}+C T^{\epsilon}\left(2 C\left\|u_{0}\right\|_{H^{s}(\mathbf{R})}\right)^{3} \leq 2 C\left\|u_{0}\right\|_{H^{s}(\mathbf{R})}
$$


thus, $\Phi(u)$ is a mapping on $B$. By using a proof similar to (4.4), by choosing $T$ sufficiently small such that $24 C^{3} T^{\epsilon}\left\|u_{0}\right\|_{H^{s}}^{2}<1$, we obtain

$$
\begin{aligned}
& \left\|\Phi\left(u_{1}\right)-\Phi\left(u_{2}\right)\right\|_{X_{s, \frac{1}{2}+\epsilon}} \\
& \quad \leq C T^{\epsilon}\left[\left\|u_{1}\right\|_{X_{s, \frac{1}{2}+\epsilon}}^{2}+\left\|u_{1}\right\|_{X_{s, \frac{1}{2}+\epsilon}+\epsilon}\left\|u_{2}\right\|_{X_{s, \frac{1}{2}+\epsilon}}+\left\|u_{2}\right\|_{X_{s, \frac{1}{2}+\epsilon}}^{2}\right]\left\|u_{1}-u_{2}\right\|_{X_{s, \frac{1}{2}+\epsilon}} \\
& \quad \leq \frac{1}{2}\left\|u_{1}-u_{2}\right\|_{X_{s, \frac{1}{2}+\epsilon}}
\end{aligned}
$$

thus, $\Phi(u)$ is a contraction mapping on the closed ball $B$. Consequently, $\Phi$ have a fixed point $u$ and the Cauchy problem for (1.1) possesses a local solution on $[-T, T]$. The uniqueness of the solution is obvious.

This completes the proof of Theorem 1.1.

\section{Proof of Theorem 1.2}

In this section, inspired by [5, 35, 45], we present the proof of Theorem 1.2. We will prove Theorem 1.2 by contradiction.

We assume that the solution map of (1.4), (1.5) is $C^{3}$ in $H^{s}(\mathbf{R})$ with $s<\frac{1}{4}$. Then, from Theorem 3 of [35], we have

$$
\sup _{t \in[0, T]}\left\|B_{3}\left(u_{0}\right)\right\|_{H^{s}} \leq C\left\|u_{0}\right\|_{H^{s}}^{3}
$$

for $u_{0} \in H^{s}(\mathbf{R})$. Here

$$
\begin{aligned}
& B_{1}\left(u_{0}\right)=e^{-t\left(-\partial_{x}^{3}-\partial_{x}^{-1}\right)} u_{0}, \\
& B_{3}\left(u_{0}\right)=\frac{1}{3} \int_{0}^{t} e^{-(t-\tau)\left(-\partial_{x}^{3}-\partial_{x}^{-1}\right)} \partial_{x}\left(\left(B_{1}\left(u_{0}\right)\right)^{3}\right) d \tau .
\end{aligned}
$$

We consider the initial data

$$
u_{0}(x)=r^{-\frac{1}{2}} N^{-s}\left\{e^{i N x} \int_{0}^{r} e^{i x \xi} d \xi+e^{-i N x} \int_{r}^{2 r} e^{i x \xi} d \xi\right\}, \quad r^{2} N=O(1), N \geq 2 .
$$

By using a direct computation, we have

$$
\mathcal{F}_{x} u_{0}(\xi)=\operatorname{Cr}^{-\frac{1}{2}} N^{-s}\left\{\chi_{[-N,-N+r]}(\xi)+\chi_{[N+r, N+2 r]}(\xi)\right\}
$$

Here $\chi_{I}$ denotes the characteristic function of a set $I \subset \mathbf{R}$. Obviously,

$$
\left\|u_{0}\right\|_{H^{s}(\mathbf{R})} \sim 1 .
$$

We define $I_{1}:=[-N,-N+r]$ and $I_{2}:=[N+r, N+2 r]$ and $\Omega_{1}:=I_{1} \cup I_{2}$. By using a direct computation, we have

$$
\mathcal{F}_{x} B_{1} u_{0}(\xi)=C e^{i t \phi(\xi)} \mathcal{F}_{x} u_{0}(\xi)
$$


Combining (5.6) with the definition of $B_{3}\left(u_{0}\right)$, we have

$$
B_{3}\left(u_{0}\right)(x, t)=C g \text {. }
$$

Here

$$
g=C r^{-\frac{3}{2}} N^{-3 s} \int_{\xi_{1} \in \Omega_{1}} \int_{\xi_{2} \in \Omega_{1}} \int_{\xi_{3} \in \Omega_{1}}\left(\sum_{j=1}^{3} \xi_{j}\right) e^{i x \sum_{j=1}^{3} \xi_{j}} H\left(\xi_{1}, \xi_{2}, \xi_{3}\right) d \xi_{1} d \xi_{2} d \xi_{3}
$$

where

$$
H\left(\xi_{1}, \xi_{2}, \xi_{3}\right)=\frac{e^{i t\left(\phi\left(\xi_{1}\right)+\phi\left(\xi_{2}\right)+\phi\left(\xi_{3}\right)\right)}-e^{i t \phi\left(\sum_{j=1}^{3} \xi_{j}\right)}}{\phi\left(\xi_{1}\right)+\phi\left(\xi_{2}\right)+\phi\left(\xi_{3}\right)-\phi\left(\sum_{j=1}^{3} \xi_{j}\right)} .
$$

We define

$$
\theta_{1}:=\phi\left(\xi_{1}\right)+\phi\left(\xi_{2}\right)+\phi\left(\xi_{3}\right)-\phi\left(\sum_{j=1}^{3} \xi_{j}\right) .
$$

From Lemma 2.4, we have

$$
\theta_{1}=-3\left[\left(\xi_{1}+\xi_{2}\right)\left(\xi_{1}+\xi_{3}\right)\left(\xi_{2}+\xi_{3}\right)\right]\left[1-\frac{1}{3 \prod_{j=1}^{3} \xi_{j}\left(\sum_{j=1}^{3} \xi_{j}\right)}\right] .
$$

To estimate $\|g\|_{H^{s}(\mathbf{R})}$, we need to consider the following three cases:

Case 1: $\xi_{j} \in I_{1} \quad(j=1,2,3)$,

Case $2: \quad \xi_{j} \in I_{1} \quad(j=1,2,3)$,

Case 3: $\xi_{j} \in I_{1} \quad(j=1,2), \quad \xi_{3} \in I_{2} \quad$ or $\quad \xi_{1} \in I_{1}, \quad \xi_{j} \in I_{2} \quad(j=2,3)$

$$
\text { or } \quad \xi_{j} \in I_{2} \quad(j=1,2), \quad \xi_{3} \in I_{1} \quad \text { or } \quad \xi_{1} \in I_{2}, \quad \xi_{j} \in I_{1} \quad(j=2,3) \text {. }
$$

We assume that $\|g\|_{H^{s}(\mathbf{R})}$ corresponding to cases 1, 2, 3 are denoted by $L_{1}, L_{2}, L_{3}$, respectively.

Case 1. In this case, we have $\left|\theta_{1}\right| \sim N^{3}$ and $\left|\xi_{1}+\xi_{2}+\xi_{3}\right| \sim N$. Since $r^{2} N=O(1)$, we have

$$
L_{1} \leq C r^{-\frac{3}{2}} N^{-3 s} N^{s} r^{\frac{5}{2}} N^{-2} \leq C N^{-2 s-\frac{5}{2}}
$$

Case 2. In this case, we have $\left|\theta_{1}\right| \sim N^{3}$ and $\left|\xi_{1}+\xi_{2}+\xi_{3}\right| \sim N$. Since $r^{2} N=O(1)$, we have

$$
L_{2} \leq C r^{-\frac{3}{2}} N^{-3 s} N^{s} r^{\frac{5}{2}} N^{-2} \leq C N^{-2 s-\frac{5}{2}}
$$

Case 3. In this case, we have $\left|\theta_{1}\right| \sim r^{2} N$ and $\left|\xi_{1}+\xi_{2}+\xi_{3}\right| \sim N$ as well as $H \leq|t|$. Since $r^{2} N=O(1)$, we have

$$
L_{3} \geq C|t| r^{-\frac{3}{2}} N^{-3 s} N^{s} r^{\frac{5}{2}} N \geq C|t| N^{-2 s+\frac{1}{2}} .
$$


Combining (5.1), (5.5) with (5.12)-(5.14), we have

$$
|t| N^{-2 s+\frac{1}{2}} \leq L_{3}-L_{1}-L_{2} \leq \sup _{t \in[0, T]}\left\|B_{3}\left(u_{0}\right)\right\|_{H^{s}} \leq C\left\|u_{0}\right\|_{H^{s}}^{3} \sim C
$$

For fixed $t>0$, when $s<\frac{1}{4}$, let $N \longrightarrow \infty$, we have $|t| N^{-2 s+\frac{1}{2}} \longrightarrow+\infty$, and this contradicts (5.15).

This ends the proof of Theorem 1.2.

\section{Acknowledgements}

The second author is supported by the Young core Teachers Program of Henan Normal University and 15A110033.

\section{Competing interests}

The authors declare that they have no competing interests.

\section{Authors' contributions}

All authors read and approved the final manuscript.

\section{Author details}

${ }^{1}$ School of Mathematics and Statistics, Lanzhou University, LanZhou, Gansu 730000, P.R. China. ${ }^{2}$ College of Mathematics and Information Science, Henan Normal University, Xinxiang, Henan 453007, P.R. China.

\section{Publisher's Note}

Springer Nature remains neutral with regard to jurisdictional claims in published maps and institutional affiliations.

Received: 8 June 2017 Accepted: 9 December 2017 Published online: 21 December 2017

\section{References}

1. Galkin, VN, Stepanyants, YA: On the existence of stationary solitary waves in a rotating fluid. J. Appl. Math. Mech. 55, 939-943 (1991)

2. Leonov, A: The effect of the Earth's rotation on the propagation of weak nonlinear surface and internal long oceanic waves. Ann. N.Y. Acad. Sci. 373, 150-159 (1981)

3. Shrira, V: Propagation of long nonlinear waves in a layer of a rotating fluid. Izv., Atmos. Ocean. Phys. 17, 76-81 (1981)

4. Shrira, V: On long essentially non-linear waves in a rotating ocean. Izv., Atmos. Ocean. Phys. 22, 395-405 (1986)

5. Bourgain, J: Periodic Korteweg de Vries equation with measures as initial data. Sel. Math. New Ser. 3, 115-159 (1997)

6. Colliander, J, Keel, M, Staffilani, G, Takaoka, H, Tao, T: Sharp global well-posedness for KdV and modified KdV on $R$ and T. J. Am. Math. Soc. 16, 705-749 (2003)

7. Guo, ZH: Global well-posedness of the Korteweg-de Vries equation in $H^{-3 / 4}$ (R). J. Math. Pures Appl. 91, $583-597$ (2009)

8. Kenig, CE, Ponce, G, Vega, L: The Cauchy problem for the Korteweg-de Vries equation in Sobolev spaces of negative indices. Duke Math. J. 71, 1-21 (1993)

9. Kenig, CE, Ponce, G, Vega, L: Well-posedness and scattering results for the generalized Korteweg-de Vries equation via the contraction principle. Commun. Pure Appl. Math. 46, 527-620 (1993)

10. Kenig, CE, Ponce, G, Vega, L: On the ill-posedness of some canonical dispersive equations. Duke Math. J. 106, 617-633 (2001)

11. Kishimoto, N: Well-posedness of the Cauchy problem for the Korteweg-de Vries equation at the critical regularity. Differ. Integral Equ. 22, 447-464 (2009)

12. Ostrovskii, LA: Nonlinear internal waves in a rotating ocean. Okeanologia 18,181-191 (1978)

13. Benilov, ES: On the surface waves in a shallow channel with an uneven bottom. Stud. Appl. Math. 87, 1-14 (1992)

14. Gilman, OA, Grimshaw, R, Stepanyants, YA: Approximate and numerical solutions of the stationary Ostrovsky equation. Stud. Appl. Math. 95, 115-126 (1995)

15. Grimshaw, R: Evolution equations for weakly nonlinear long internal waves in a rotating fluid. Stud. Appl. Math. 73, 1-33 (1985)

16. Levandosky, S, Liu, Y: Stability of solitary waves of a generalized Ostrovsky equation. SIAM J. Math. Anal. 38, $985-1011$ (2006)

17. Liu, Y, Varlamov, V: Stability of solitary waves and weak rotation limit for the Ostrovsky equation. J. Differ. Equ. 203 159-183 (2004)

18. Levandosky, S: On the stability of solitary waves of a generalized Ostrovsky equation. Anal. Math. Phys. 2, 407-437 (2012)

19. Gui, GL, Liu, Y: On the Cauchy problem for the Ostrovsky equation with positive dispersion. Commun. Partial Differ. Equ. 32, 1895-1916 (2007)

20. Guo, BL, Huo, ZH: The global attractor of the damped forced Ostrovsky equation. J. Math. Anal. Appl. 329, 392-407 (2007)

21. Huo, Z, Jia, YL: Low-regularity solutions for the Ostrovsky equation. Proc. Edinb. Math. Soc. 49, 87-100 (2006)

22. Isaza, P: Unique continuation principle for the Ostrovsky equation with negative dispersion. J. Differ. Equ. 225 796-811 (2013) 
23. Isaza, P, Mejía, J: Cauchy problem for the Ostrovsky equation in spaces of low regularity. J. Differ. Equ. 230, 661-681 (2006)

24. Isaza, P, Mejía, J: Global Cauchy problem for the Ostrovsky equation. Nonlinear Anal. TMA 67, 1482-1503 (2007)

25. Isaza, P, Mejía, J: Local well-posedness and quantitative ill-posedness for the Ostrovsky equation. Nonlinear Anal. TMA 70, 2306-2316 (2009)

26. Isaza, P, Mejía, J: On the support of solutions to the Ostrovsky equation with negative dispersion. J. Differ. Equ. 247 1851-1865 (2009)

27. Linares, F, Milanés, A: Local and global well-posedness for the Ostrovsky equation. J. Differ. Equ. 222, 325-340 (2006)

28. Molinet, L: Sharp ill-posedness results for the KdV and mKdV equations on the torus. Adv. Math. 230, 1895-1930 (2012)

29. Varlamov, V, Liu, Y: Cauchy problem for the Ostrovsky equation. Discrete Contin. Dyn. Syst., Ser. A 10, 731-753 (2004)

30. Wang, H, Cui, S: Well-posedness of the Cauchy problem of Ostrovsky equation in anisotropic Sobolev spaces. J. Math. Anal. Appl. 327, 88-100 (2007)

31. Tsugawa, K: Well-posedness and weak rotation limit for the Ostrovsky equation. J. Differ. Equ. 247, 3163-3180 (2009)

32. Coclite, GM, di Ruvo, L: Convergence of the Ostrovsky equation to the Ostrovsky-Hunter one. J. Differ. Equ. 256 3245-3277 (2014)

33. Coclite, GM, di Ruvo, L: Dispersive and diffusive limits for Ostrovsky-Hunter type equations. Nonlinear Differ. Equ. Appl. 22, 1733-1763 (2015)

34. Li, YS, Huang, JH, Yan, W: The Cauchy problem for the Ostrovsky equation with negative dispersion at the critical regularity. J. Differ. Equ. 259, 1379-1408 (2015)

35. Bejenaru, I, Tao, T: Sharp well-posedness and ill-posedness results for a quadratic non-linear Schrödinger equation. J. Funct. Anal. 233, 228-259 (2006)

36. Rauch, J, Reed, M: Nonlinear microlocal analysis of semilinear hyperbolic systems in one space dimension. Duke Math. J. 49, 397-475 (1982)

37. Beals, M: Self-spreading and strength of singularities for solutions to semilinear wave equations. Ann. Math. 118, $187-214(1983)$

38. Bourgain, J: Fourier transform restriction phenomena for certain lattice subsets and applications to nonlinear evolution equations, part I: Schrödinger equations. Geom. Funct. Anal. 3, 107-156 (1993)

39. Bourgain, J: Fourier transform restriction phenomena for certain lattice subsets and applications to nonlinear evolution equations, part II: the KdV equation. Geom. Funct. Anal. 3, 209-262 (1993)

40. Klainerman, S, Machedon, M: Smoothing estimates for null forms and applications. Int. Math. Res. Not. 1994(9), 383-389 (1994)

41. Kenig, CE, Ponce, G, Vega, L: A bilinear estimate with applications to the KdV equation. J. Am. Math. Soc. 9, 573-603 (1996)

42. Ionescu, AD, Kenig, CE, Tataru, D: Global well-posedness of the KP-I initial-value problem in the energy space. Invent. Math. 173, 265-304 (2008)

43. Li, YS, Wu, YF: Global well-posedness for the Benjamin-Ono equation in low regularity. Nonlinear Anal. 73, 1610-1625 (2010)

44. Grünrock, A: New applications of the Fourier restriction norm method to wellposedness problems for nonlinear evolution equations. Ph.D. dissertation, Universität Wuppertal (2002)

45. Tzvetkov, N: Remark on the local ill-posedness for KdV equation. C. R. Math. Acad. Sci. Paris, Sér. I 329, 1043-1047 (1999)

\section{Submit your manuscript to a SpringerOpen ${ }^{\circ}$ journal and benefit from:}

- Convenient online submission

- Rigorous peer review

- Open access: articles freely available online

- High visibility within the field

- Retaining the copyright to your article

Submit your next manuscript at $\gg$ springeropen.com 